\title{
Spectroscopic characterisation of the PSI-2 plasma in the ionising and recombining state
}

\author{
A. Pospieszczyk ${ }^{a, *, 1}$, M. Reinhart ${ }^{a}$, B. Unterberg ${ }^{a}$, S. Brezinsek ${ }^{a}$, A. Kreter ${ }^{a}$, U. Samm $^{\text {a }}$, G. Sergienko $^{\mathrm{a}}$, \\ B. Schweer ${ }^{\text {a }}$, C. Salmagne ${ }^{a}$, D. Reiter ${ }^{a}$, M. Baelmans ${ }^{b}$, D. Wünderlich ${ }^{c}$, U. Fantz $^{\mathrm{d}}$ \\ a Institute of Energy and Climate Research - Plasma Physics, Forschungszentrum Jülich GmbH, Association EURATOM-FZJ, Partner in the Trilateral Euregio Cluster, \\ D-52425 Jülich, Germany \\ ${ }^{\mathrm{b}}$ Department of Mechanical Engineering, K.U. Leuven, Celestijnenlaan 300A, 3001 Heverlee, Belgium \\ ${ }^{\mathrm{c}}$ Max-Planck-Institute für Plasmaphysik, EURATOM Association, Boltzmannstr. 2, D-85748 Garching, Germany \\ d Experimentelle Plasmaphysik, Augsburg University, Universitätsstr. 1, D-86135 Augsburg, Germany
}

\begin{abstract}
A B S T R A C T
In the linear plasma simulator PSI 2, capable of producing electron temperatures and densities of $120 \mathrm{eV}$ and $10^{17} 10^{19} \mathrm{~m}^{3}$ in the target region, a deuterium plasma with a simultaneous ionising and recombin ing region has been produced. The latter appeared when a sufficiently high extra deuterium gas flow was applied at the target region. An imaging spectrometer was used to measure $T_{\mathrm{e}}$ and $n_{\mathrm{e}}$ profiles via the analysis of the Paschen series resulting in drops down to $0.1 \mathrm{eV}$ and $2 \times 10^{18} \mathrm{~m}^{3}$ in the recombining region whereas intensity ratios of $\mathrm{H} \alpha, \mathrm{H} \beta$ and $\mathrm{H} \gamma$ provided $n_{\mathrm{e}}$ in the ionising part. In the latter case $n_{\mathrm{e}}$ was also deduced from the rotational temperature of the $\mathrm{D}_{2}$ molecules. Comparison with probe data yielded a reasonable agreement in that case. A detailed analysis of the atomic level populations and their light emissions provided some insight into the contribution of possible recombination mechanisms such as EIR and MAR.
\end{abstract}

\section{Introduction}

The investigation of plasmas under divertor like conditions is of utmost importance for fusion experiments. Here the interaction of the particles with material components takes place and determines both the impurity content of the main plasma and the exhaust of the helium produced. In order to study the underlying processes and under well defined conditions in detail, several linear plasma machines have been built (PISCES A\&B [1], NAGDIS I\&II [2], PSI $1 \& 2$ [3], PILOT PSI [4]), or have just finished construction (MAG NUM PSI [5]).

The PSI 2 plasma simulator has been used for a decade at the Humboldt University in Berlin and is now back in operation at the IEK 4 (plasma physics) of the Research Centre Jülich (FZJ). There it now acts as a pilot experiment for the JULE PSI facility, a linear plasma device in a hot cell environment for experiments with neutron activated, plasma facing materials and beryllium. For JULE PSI, spectroscopy is a favourable method to measure plas ma parameters as it has big advantages compared to movable mechanical systems such as Langmuir probes. Moreover, spec trometers can be placed outside the hot cell environment. In this

\footnotetext{
* Corresponding author.

E-mail address: a.pospieszczyk@fz-juelich.de (A. Pospieszczyk).

1 Presenting author.
}

contribution, the aim is, therefore, twofold. We report on first mea surements to determine the plasma characteristics by spectros copy in PSI 2 and to assess this method for application in a hot cell. Secondly a special recombining plasma state can be produced, which also often occurs in the inner divertor of a tokamak, and which has already been extensively studied when the experiment was located in Berlin [6,7]. Here, the spectroscopic capabilities, being developed for the mentioned, next generation of linear plas ma machines, are demonstrated by their application to the study of this special plasma state.

\section{Experiment}

PSI 2 (Fig. 1) produces a plasma column of $2.5 \mathrm{~m}$ length and about $6 \mathrm{~cm}$ diameter in an axial magnetic field of $0.1 \mathrm{~T}$. In the tar get region, the temperature and density reach values of about 1 $20 \mathrm{eV}$ and $10^{17} 10^{19} \mathrm{~m}^{3}$. A recombining deuterium plasma was produced with discharge currents and voltages of $50 \mathrm{~A}$ and $50 \mathrm{~V}$. In addition to the gas flow of deuterium into the plasma source a constant deuterium gas flow into the target region was applied to increase the neutral pressure there up to $10^{2}$ mbar. Under these conditions, a recombining zone develops in the centre of the plas ma column because of the hollow electron temperature profile due to the hollow cathode in the source region of the plasma while the hotter edge of the plasma remains in an ionising state. The 


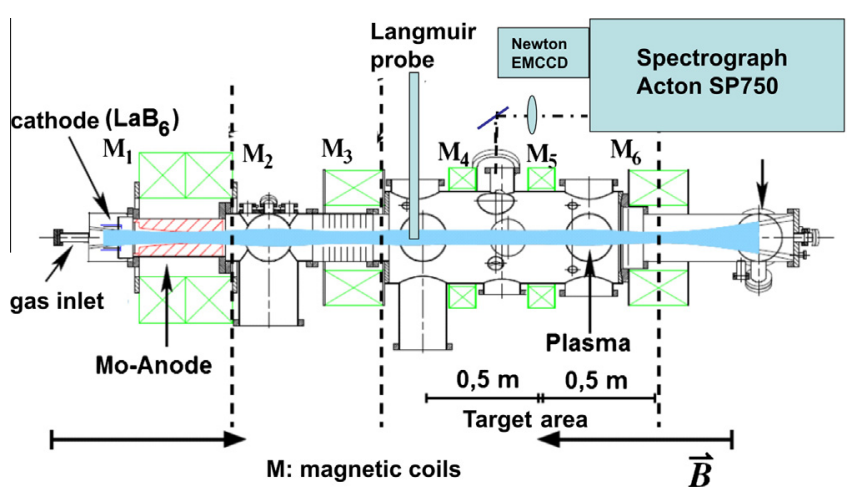

Fig. 1. Outline of PSI-2.

presence of the recombining state is characterised by a change of the plasma colour from the red into the blue caused by the prefer ential population of higher order Balmer line levels.

The major optical diagnostic is also sketched in Fig. 1 and con sists of a 2D Newton EMCD detector coupled to an ACTON SP750 spectrometer. For standard electron temperature and density pro file measurements a Langmuir probe has been used which also scans the plasma column radially. It should specifically be noted here that the probe and the optical diagnostics do not share the same observation flange. In fact the probe is positioned about $0.4 \mathrm{~m}$ upstream which may explain some differences described in the subsequent section.

\section{Results and discussion}

In the experiments performed a basic plasma was created with a source current of $50 \mathrm{~A}$; the neutral pressure in the target chamber was $0.3 \times 10^{3}$ mbar. This discharge current is nearly one order of magnitude smaller than the one used in [7] and may explain some differences in the plasma parameters (see below). Additional $D_{2}$ gas flows up to $300 \mathrm{sccm}$ were applied in the target region, which increased the total neutral pressure in five nearly equal steps from $0.6 \times 10^{2}$ mbar to $2.2 \times 10^{2}$ mbar. A remarkable recombi nation effect starts at a neutral pressure of $0.9 \times 10^{2}$ mbar and leads to a drop of the $D_{\alpha} / D_{\gamma}$ ratio by a factor of 20 which is an indicator of a preferential population of the higher levels.

The corresponding probe measurements can be seen in Fig. 2 . They show the radial electron temperature and density profiles in the upper part of the discharge, which also contains the recom bining plasma from about $02.5 \mathrm{~cm}$. In our case both temperature and density show the usual hollow pattern in PSI 2 which reflects the structure of the electron emitting cathode cylinder in the source. However, for high values the density profile can form a bell shape whereas the temperature profiles remains always hollow. The temperature in the (ionising) region falls continuously whereas the density develops a maximum at $0.9 \times 10^{2} \mathrm{mbar}$ and stays then more or less constant. However, the values around $1 \mathrm{~cm}$ (in the recombining region) do not yet fully reflect the tem perature data found there by spectroscopy.

An example of the spectroscopic capabilities can be seen in Figs. 3 and 4 where for a recombining case spectra at a neutral pressure of $1.3 \times 10^{2}$ mbar three wavelength ranges are dis played. The atomic lines of the Paschen series (around $900 \mathrm{~nm}$ ) and of the Balmer series (around $380 \mathrm{~nm}$ ) show clearly the en hanced emission from the high $n$ levels whereas the $D_{2}$ molecular Fulcher $\alpha$ spectrum is not affected in the range of the recombining zone.

Therefore, in order to evaluate the plasma parameters by emis sion spectroscopy the methods have to be adapted differently in
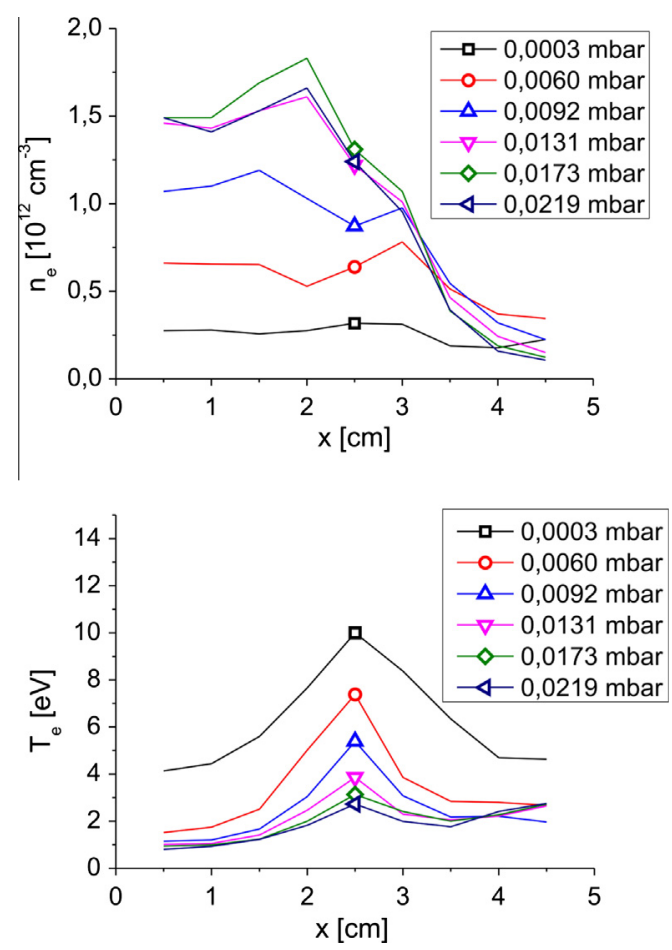

Fig. 2. Probe measurements for different neutral pressures.

the recombining and in the ionising zone. The respective atomic data for both the exciting and recombining processes of the hydro gen atom have been compiled from [8]. In the ionising region the intensity ratios of $\mathrm{H} \alpha, \mathrm{H} \beta$ and $\mathrm{H} \gamma$ have served for the determina tion of the electron density. A comparison of the values deduced with the respective data from the probe can be found in Fig. 5. The "optical" data are always larger and show a slightly steeper trend with growing neutral density. As expected, larger deviations occur from the onset of the recombination at $0.9 \times 10^{2}$ mbar and the change in the level population mechanism.

Recently a way to deduce the electron density from the molec ular properties of the hydrogen molecule has been pointed out [9]. There, it is has been shown that for plasmas with electron temper atures above $15 \mathrm{eV}$ a linear dependence exists between the rota tional temperature of the Fulcher bands and the electron density. This dependence, found on the tokamak TEXTOR, has been adapted to the conditions of PSI 2 and the respective densities were de duced from the spectra shown in Fig. 4. These data can also be found in Fig. 5, however, the agreement especially for the non recombining case is not (yet) satisfactory. Running calibration experiments under PSI 2 conditions seem to lead to much better results and will be published in a forthcoming paper.

In the recombining region we used the intensity ratios of high $n$ atomic hydrogen lines to compute the electron temperature. The Paschen series is preferably suited for this purpose because it pro vides a broader variation range than the Balmer series. Similar ap proaches have already been reported in [10]. Here the ratio $\mathrm{P}_{9->3} /$ $P_{7->3}$ has been used to determine $T_{\mathrm{e}}$ as a function of the neutral pressure (see Fig. 6), which shows that the temperature in the recombining zone finally reaches values of $0.1 \mathrm{eV}$ at the highest neutral pressures of about $2.0 \times 10^{2}$ mbar. However, the probe values do not agree with this finding probably again a result of its different location in the target zone, although electron temper atures in the range of $0.5 \mathrm{eV}$ can in principle be measured by probes (see [7]).

We also investigated the possibility of using Stark broadening to evaluate the electron density in this region. This method is well 

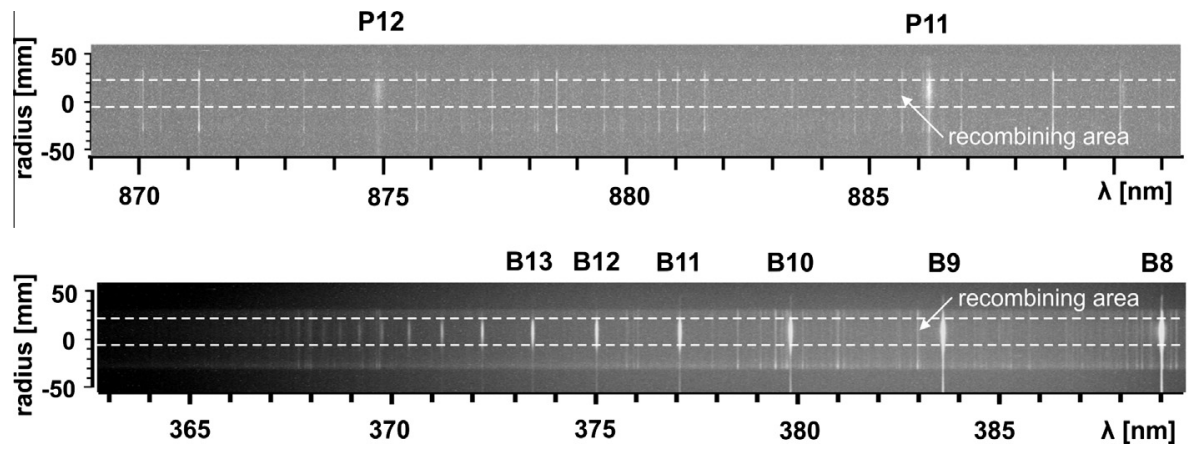

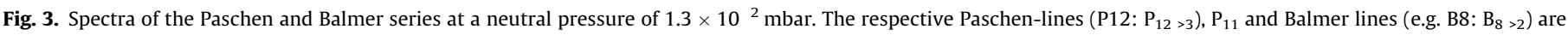
indicated.

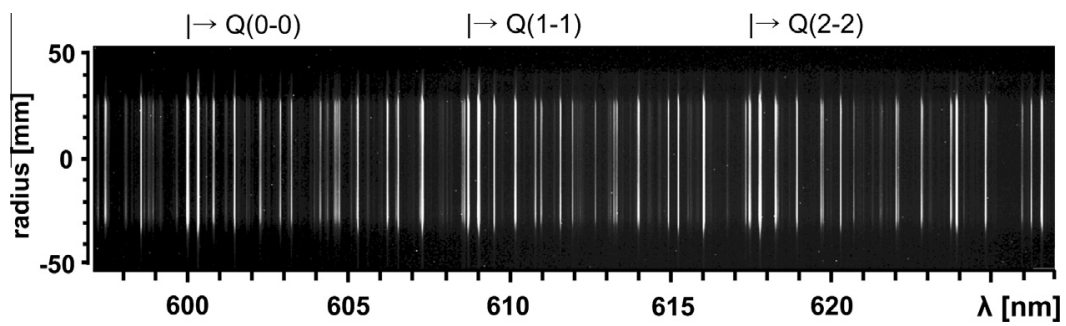

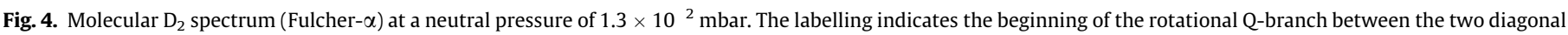
vibrational levels 0,1 , and 2 .

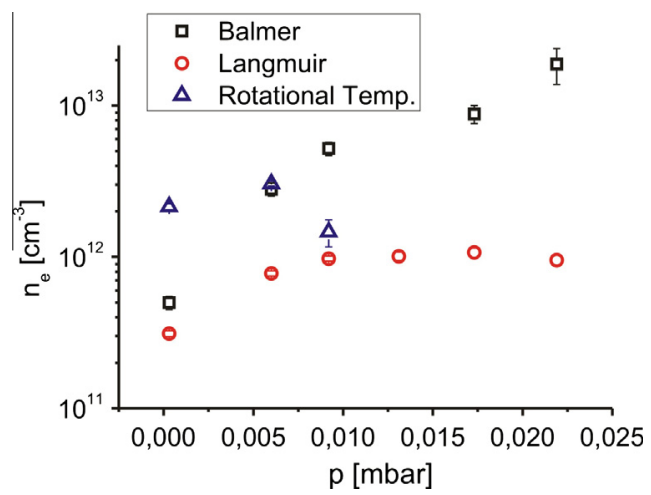

Fig. 5. Comparison of the electron densities in the ionising zone obtained by different methods.

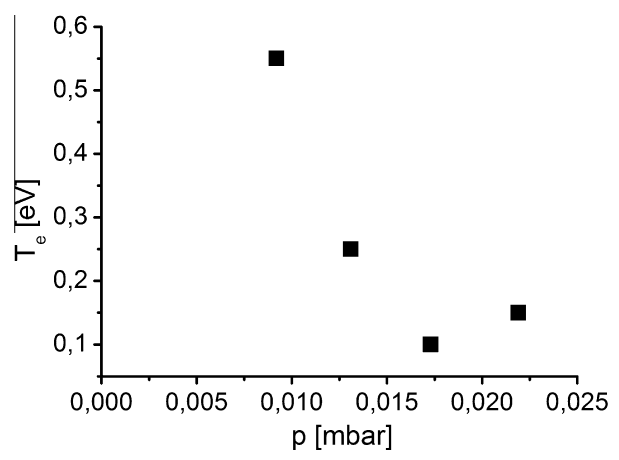

Fig. 6. Electron temperatures in the recombining zone for different neutral pressures as obtained by the Paschen series ratio $\mathrm{P}_{9>3} / \mathrm{P}_{7}>3$.

known for the diagnostics of recombining plasmas and its princi ples can be found in [11]. As the line width scales with the square of the wavelength it is advisable to use the lines of the Paschen in

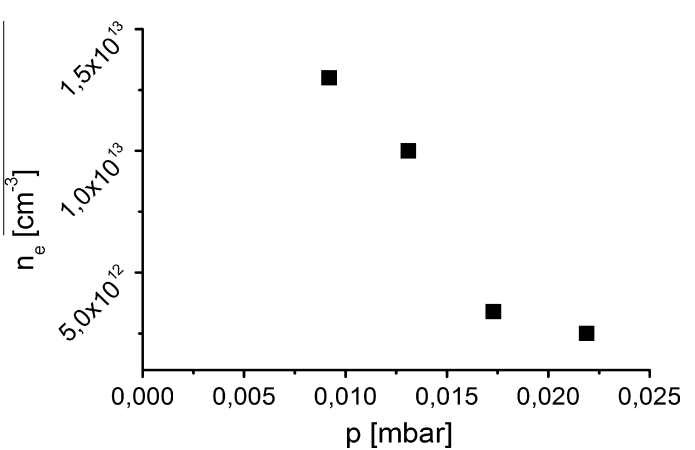

Fig. 7. Electron densities in the recombining zone for different neutral pressures as obtained by evaluation of the Stark broadening of the $\mathrm{P}_{10>3}$ line.

stead of those from the Balmer series. For our evaluation of the $\mathrm{P}_{10->3}$ line we have used the data from [12]. The results can be seen in Fig. 7 and show a constant decrease of the electron densities down to values of $10^{12} \mathrm{~cm}^{3}$ which can, of course, be expected at these extremely low temperatures.

In order to obtain a deeper insight into the underlying recombi nation mechanisms plots of the emission from the individual atomic levels have been produced. As an example the case with the largest gas puff $(0.0219$ mbar $)$ for the centre of the recombin ing region is shown (Fig. 8). In a recombining plasma more higher $n$ levels can be measured. Therefore, this regime can be used for a more detailed analysis of the individual mechanisms. The popula tion of the higher $n$ in the recombining zone by EIR (Electron In duced Recombination) is quite obvious. However, the lower $n$ can

in the presence of a high molecular density also be populated by MAR (Molecular Assisted Recombination) see e.g. [13]. Addi tionally shown in Fig. 8 are model calculations using the Yacora code [14], a new collisional radiative model for low pressure, low temperature hydrogen plasmas, including six possible excitation channels. This comparison can serve to find the individual ionic 


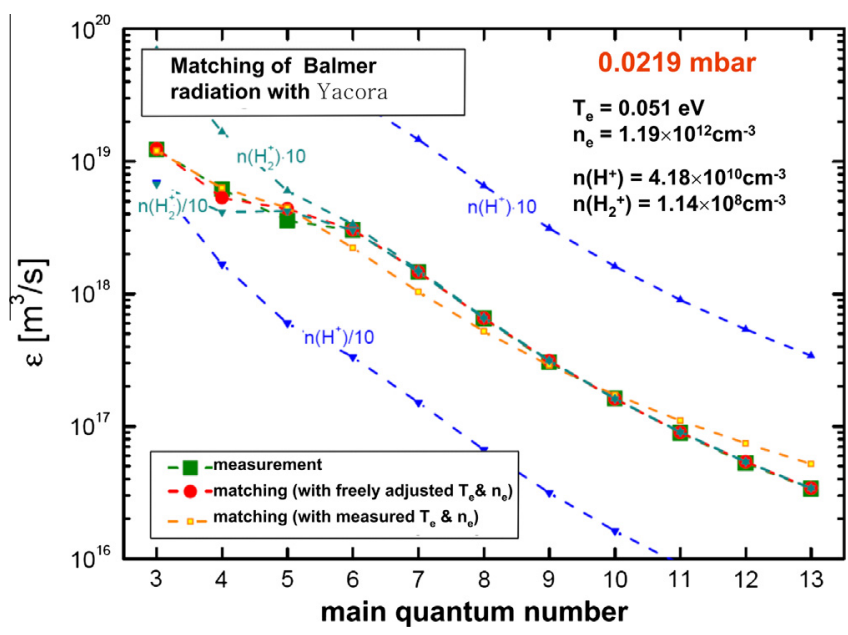

Fig. 8. Local emission from the different atomic Balmer levels for a high neutral pressure (0.0219 mbar) in the recombing zone and comparison with modelling. The sensitivity of the Yacora code used [14] is shown by a variation of the ionic species $\mathrm{H}^{+}$and $\mathrm{H}_{2}^{+}$by one order of magnitude in both directions. These amount to $3.5 \%$ and $10^{4}$ of the electron density respectively. 0-D Yacora code matching then requires postulation of dominant $\mathrm{H}_{3}^{+}$as positive charge carrier.

fractions and actually one can see from the variations of the code input (changing the $\mathrm{H}^{+}$and $\mathrm{H}_{2}^{+}$by two orders of magnitude) that it is a quite sensitive method.

\section{Conclusions and outlook}

For the diagnostics of low temperature deuterium boundary plasmas, optical spectroscopy of the atomic and molecular species can be an adequate and easy means for the determination of basic plasma parameters. Moreover, the detailed investigation of the atomic and molecular level populations provides a profound in sight into the discharge conditions, which can help to adjust the load on fusion relevant targets in the beam. With this knowledge one could both reduce the necessary access to devices in a hot cell environment and a failure of additional mechanical components (e.g. probes). Whereas for a recombining plasma the determination of electron temperature and density via the Balmer and Paschen line diagnostics is a well and easily available mean, an optical method for an ionising plasma is only available for the (high) elec tron density. However, this problem could probably be overcome by the simultaneous use of tracer impurities e.g. helium. For the latter considerable experiences exist already for both tokamak [15] and plasma simulator applications [16]. Its potential for dis charges in PSI 2 will be further investigated as it offers a possibility to become independent of the type of working gas.

\section{References}

[1] R.P. Doerner et al., Phys. Scr. T111 (2004) 75

[2] N. Ohno, N. Ezumi, S. Takamura, S.I. Kraheninnikov, A.Yu. Pigarov, Phys. Rev. Lett. 81 (1998) 818-821.

[3] H. Kastelewicz, G. Fussmann, Contrib. Plasma Phys. 44 (2004) 352-360.

[4] G.J. van Rooij, V.P. Veremiyenko, W.J. Goedheer, B. de Groot, A.W. Kleyn, P.H.M. Smeets, T.W. Versloot, D.G. Whyte, R. Engeln, D.C. Schram, N.J. Lopes Cardozo, Appl. Phys. Lett. 90 (2007) 121501

[5] J. Rapp, W.R. Koppers, H.J.N. Van Eck, G.J. Van Rooij, W.J. Goedheer, B. De Groot, R. Al, M.F. Graswinckel, M.A. Van Den Berg, O. Kruyt, Fusion Eng. Des. 85 (2010) 1455-1459.

[6] U. Wenzel, D. Schröder, W. Bohmeyer, G. Fussmann, IEEE Tran. Plasma Sci. 33 (2005) 366-367.

[7] U. Wenzel, D. Schröder, G. Fussmann, Contrib. Plasma Phys. 47 (2007) 451 457.

[8] Atomic Data Analysis Structure (ADAS), <http://adas.phys.strath.ac.uk>.

[9] S. Brezinsek, Jül-Bericht 3962, Forschungszentrum Jülich, Jülich, 2002. ISSN 0944-2952;

A. Pospieszczyk, Nuclear Fusion Research: Understanding Plasma-Surface Interaction, vol. 78, Springer Series in Chemical Physics, (2005) 135.

[10] A.G. Meigs, W. Fundamenski, C. Jupen, A. Larsen, S. Loch, M. O'Mullane, H.P. Summers, in: 27th EPS Conference on Contr. Fusion and Plasma Phys. Budapest, ECA, vol. 25A, 2000.

[11] H.R. Griem, Principles of Plasma Spectroscopy, Cambridge University Press, 1997.

[12] C. Stehle, R. Hutcheon, Astron. Astrophys. Suppl. Ser. 140 (1999) 93.

[13] K.J. Gibson, P.K. Browning, B. Mihaljcic, D.A. Forder, J. Hugill, J. Nuclear Mater. 313-316 (2003) 1253-1257.

[14] D. Wünderlich, S. Dietrich, U. Fantz, J. Quant. Spectrosc. Radiat. Transfer 110 (2009) 62.

[15] O. Schmitz, I.L. Beigman, L.A. Vainshtein, B. Schweer, M. Kantor, A. Pospieszczyk, Y. Xu, M. Krychowiak, M. Lehnen, U. Samm, B. Unterberg, the TEXTOR team, Plasma Phys. Controlled Fusion 50 (2008) 115004.

[16] S. Kajita, D. Nishijima, E.M. Hollmann, N. Ohno, Phys. Plasmas 16 (2009) 063303. 